\title{
Enhancement of Images Taken in Fog Condition: A Review
}

\author{
Rajashree Das, Tridipjit Rajkonwar, Dr. Dibya jyoti Bora \\ Department of Information Technology \\ School of Computing Science \\ Kaziranga University \\ Assam, India
}

\begin{abstract}
Images captured using camera systems in foggy weather conditions often suffer from poor visibility and can be seriously degraded due to atmospheric conditions, which creates a lot of impacts on the outdoor computer vision systems. To solve this problem, image enhancement is very important as this process is used for enhancing the quality of an image, and for this purpose numerous visibility enhancement techniques have been used and applied. In this paper, we have tried to describe how to enhance an image using different techniques and methods. For this, various techniques and methods have been studied for image enhancement used in different research and review papers. The main goal of this paper is to understanding and reviewing the techniques used for image enhancement.
\end{abstract}

Index Terms - atmospheric conditions, computer vision and visibility enhancement.

\section{INTRODUCTION}

$\mathrm{F}$ OG IS the visible aggregation of a great number of water droplets and ice crystals which are suspended in the atmosphere close to the ground level. These droplets and ice crystals reduces the amount of visibility and contrast of the image which results in blurring of edge information and make the object identification way too difficult. Image enhancement is the process of adjusting digital pictures in order that the results square measure a lot of appropriate for show or any image analysis. Image enhancement techniques remove blur and noise, improve the standard of quality of images for human viewing, increase distinction, as enhancement operations. It is often required to enhance the value of images and therefore, certain image enhancement techniques have been use. Enhancement of image is may be a terribly difficult issue in several analysis and applications. The technique applied for enhancing images is applicable for medical image processing and image processing application areas like satellite image processing, biometric image processing, etc.

\section{LITERATURE REVIEW}

In the past few years, many researchers have published various research and review papers on Image Enhancement techniques using different algorithms. So far, several task have been done for image enhacement. So, here in this section, some of the work have been discussed.

Ping-Juei Liu, Shi-Jinn Horng, Jzau-Sheng Lin et al. [1] proposed the paper named Contrast in Haze removal :Configurable Contrast enhancement Model Based on Dark Channel Prior. In this review paper, De-hazing is carried out by adjusting the saturation and contrast of the image to improve the quality of the post-processed image. The struggling in haze removal algorithm is reformulated subjecting to luminance reconstruction scheme which is based on the statistical analysis of luminance value of the image. The accretion of contrast is based on the variance in the gradient space and the interpretation of the contrast showing that the dark channel magnifies the divergence details by increasing the changes in the input image gradient or the saturation of the scene radiance is enhanced by decreasing the differences to the estimated initial dark channel. The final resultant contrast value imperious for the given brightness value. Here, the atmospheric light estimation module operates on colour consistancy method which outperforms even when the noise is accepted.

Zheqi Lin et al. [2] proposed "Dehazing for images and video using Guided Filter" where high-performance vision algorithm is required for the effective removing of haze and fog from the image. So here in this paper, a fast real-time image and video dehazing method has been proposed. And here also the airlight and the down-sampled transmission estimation and extraction are performed ease using this proposed algorithm. In this paper, they have used the improved guided filter is used for estimating the transmission map which can be further refined and up- sampled according to one's need.

R. Ahila Priyadarshini, S. Aruna et al. [3] proposed "Visibility Enhancement Technique for Hazy Scenes". Here in this paper, an effective visibility enhancement technique for single image de-hazing is designed by using Dark Channel Prior technique. This will helps in estimating the dark pixels having low intensity at any one of the RGB channels and this dark channel provides exact estimation for obtaining the transmission map. For the edge preservation of transmission map, the bilateral filter has been used. And to obtain the gamma correction technique and for estimating the exact colour of the hazy input image, a Laplacian distribution value is used. And for gauging the sufficient transmission map, gamma correction technique has been used. To evaluate the quality of the enhanced image, performance metric such as PSNR, e metric and $\sigma$ metric are used for measurement.

Mohammad Javed Abbaspour, Mehran Yazdi, Mohammadali Masnadi-shirazi et al.[4] proposed "A New Fast Method for Foggy Image Enhancement". In this paper, a novel method has been proposed to enhance the contrast in 
foggy images and this develops an image atmospheric model which is based on the Koschmieder's theory of atmospheric vision. To acquire an outline of a strength of the fog in different areas of the image morphological operators operation is performed. This proposed algorithm outperforms regarding quantitative and qualitative analysis and also the computation time is low.

Ashok Shrivastava, Sanjay Jain et al.[5] proposed "Single Image Dehazing based on one dimensional linear filtering and adaptive Histogram Equalization method". In this review paper, the study presents a single image de-hazing method which is based on a one-dimensional linear filter. The main goal of this proposed paper is to resolve any type of foggy issue by using this algorithm, which is basically based on mean enhancement methodology and adaptive histogram equalization method. YCbCr model excels in colour compression in which Y luminance can be used separately for storing in high resolution and the chromaticity components treated separately so that it can be used to enhance the results. Eventually, it achieves the linear complexity and results demonstrates the effectiveness of this algorithm.

S. S. Negi, Y. S Bhandari et al.[6] proposed "A Hybrid approach to Image Enhancement using Contrast Stretching on Image Sharpening and the analysis of various cases arising using Histogram", where they have discussed about the contrast stretching and image sharpening techniques. It is an approach that will concurrently adjusts contrast and enhances boundaries of the input image. On the gray-scale image, this contrast stretching will be applied and then it proceeds to Laplacian mask, and then finally, Laplacian image will be included to the original gray-scale image to obtain the desired sharpened image.

Seyed Pooya Ehsani, Hojjat Sayed Mousavi, Babak. H. Khalaj et al.[7] proposed "Chromosome Image Contrast Enhancement Using Adaptive, Iterative Histogram Matching”. Here, in this paper, they have explained an adaptive and iterative histogram matching (AIHM) algorithm for chromosome contrast enhancement used in the various medical applications. To meet the various requirements of the user and to obtain the different results, some parameters in the presented model has to be selected. The detailed simulations were accomplished by using different sets of single chromosomes, indicating that the proposed methodology enhances the details appropriately.

Che-Lun Hung, Ren-You Yan, Hsiao-His Wang et al.[8] proposed "Parallel image dehazing algorithm based on GPU using fuzzy system and Hybrid evolution algorithm" where they have explained a parallel hybrid evolution algorithm based on GPU which is proposed to enhance the computational performance. In this conventional evolution algorithm, the calculation of fitness function occupies most of the processing time. So to overcome such circumstances, we need to implement this part on GPU by using CUDA framework to reduce the processing load.

Kwang Yeon Choi, Kyeong Min Jeong, Byung Cheol Song et al.[9] proposed "Fog detection for de-fogging of road driving images".In this paper, they have generally explained the fog removal technique deteriorate the visual due to excessive contrast improvement. Here the fog detection algorithm is designed such that it selectively apply de-fogging method only at a foggy region. Apart from, an excessive contrast enhancement adjustment and luminance compensation should be done to avoid the output to be too dark. This proposed algorithm will be able to produce $97 \%$ of the fog detection accuracy and the subjective image quality is improved.

Khairunnisa Hasikin, Nor Ashidi Mat Isa et al.[10] proposed "Enhancement of the low contrast image using fuzzy set theory" where they have discussed fuzzy gray-scale enhancement technique. The fuzzy measures in the image are augmented by using the mentioned technique. By using power law transformation and saturation operator, the membership function has been modified. The intensities are increased of the underexposed regions, and intensities are decreased of the overexposed region, which is why the dynamic range is to be maintained. Other methods that are being used are INT operator and NINT operator.

Raju, Ganesamoorthy, Madhu S. Nair et al.[11] proposed "A new fuzzy-based decision algorithm for high-density impulse noise removal". In this paper, they have discussed about a new fuzzy logic and histogram-based algorithm for magnifying low contrast color image. In this methodology, there are two parameters such as $\mathrm{K}$ and $\mathrm{M}$, where $\mathrm{K}$ is the contrast identification parameter and $\mathrm{M}$ is the image's average intensity parameter. The RGB image is transformed to HSV color space. Under the controlling of parameter M, V component is strained in order to magnify the image. The quality of visuality is modified by this method.

Dong-Liang Peng, Tie-Jun Wu et al.[12] proposed "A generalized image enhancement algorithm using fuzzy sets and its application". Here, in this paper, the generalized fuzzy enhancement method that overcomes the limitations of the traditional fuzzy method of enhancement. The enhancement problem that occurs because of the low contrast and the narrow gray range images is solved by this method. Here, they have focused on the improved label algorithm is used for image segmen- tation and recognition, which is helpful in understanding an image and in object recognition.

\section{IMAGE ENHANCEMENT TECHNIQUES}

Various techniques are used for image enhancement, which are given below.

\section{A. Histogram Equalization}

Histogram equalization is a very common method in image processing of contrast adjustment using the input image's histogram. Suppose we have an image which is predominantly dark. Then its histogram would be skewed towards the lower end of the grey scale and all the image detail is compressed into the dark end of the histogram. If it could stretch out the grey levels at the dark end to build a more consistent histogram then the image would become 
much understanding. Histogram equalization stretches the histogram across the entire spectrum of pixels $(0-255)$. It increases the contrast of images for the completeness of human scrutiny and can be applied to normalize illumination variations in image understanding problems. Histogram equalization is one of the operations that can be applied to acquire new images based on histogram specification or modification. Histogram equalization is considered a global technique. This process is quite simple to understand and for each brightness level $\mathrm{j}$ in the original image, the new pixel level value $(\mathrm{k})$ is calculated as given in equation

$$
K=\sum_{i=0}^{j} N_{i} / T \ldots \ldots \ldots \ldots \ldots
$$

Here, the sum counts the number of pixels in the image with the brightness equal to or less than $\mathrm{j}$, and $\mathrm{T}$ is the total number of pixels. The main objective of histogram equalization is to find gray level transformation function $\mathrm{T}$ to transform image f such that the histogram of T(f) is equalized.

\section{B. Convolutional Neural Network}

Convolutional Neural Networks (CNN) is a class of deep learning neural networks. In short it is a machine learning algorithm that can take in an input image, assign importance to various aspects/objects in the image, and be able to differentiate one from the other. CNN algorithm works by extracting features from the images. Any CNN consists of the following:

- The Input layer which is a grayscale image.

- The Output layer which is a binary or multi-class labels.

- Hidden layers consisting of convolution layers, rectified linear unit layers, the pooling layers, and a fully connected Neural Network.

The job of CNN is to lessen the images into a form that is easier to process, without losing features critical towards a good prophecy. This is important when we need to make the algorithm scalable to massive datasets. A CNN works by extracting features from the input images. This abolish the need for manual feature extraction. The features are not trained. They are learned while the network trains on a set of images. With the help of this, one can make deep learning models extremely accurate for computer vision tasks.

\section{Fuzzy Logic Technique}

Fuzzy logic is a technique used for representing and manipulating uncertain information. This logic has been productively found in different elements of image processing. Recently fuzzy based algorithms for image enhancement have been evolved with better performance compared to that of conventional and other advanced techniques like GLG. This image processing technique includes mainly three stages, they are image fuzzification, modification of the membership values, and, if needed, image DE fuzzification. After the images data are converted from gray-level domain to the fuzzy membership realm, relevant fuzzy techniques modify the membership values.

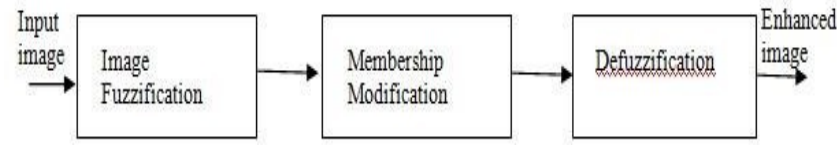

Fig.1

\section{Neuro Fuzzy System}

A Neuro-fuzzy system is a fuzzy system that uses a learning algorithm derived from or inspired by neural network theory to determine its parameters by processing data samples. These are the Artificial Neural Network (ANN) based fuzzy systems. An ANN dictate the effects of data samples by simply processing it. Prophetic power of ANN is more than compared to that of signal analysis techniques. Fuzzy set theories are essential basically for dealing with the uncertainty. Neuro-Fuzzy system is basically a system where the fuzzy rules and sets are adjusted by using neural network techniques in repetitious manner with the set of pair of input and output data vectors. At first, such system acts like a neural network where learning of parameters occurs and at the time of execution it behaves like a fuzzy.

Neural networks will detect all types of noise whether it is salt and pepper, gaussian and non-gaussian noise. And then, the Fuzzy logic will apply proper filter based on what type of noise the image has.

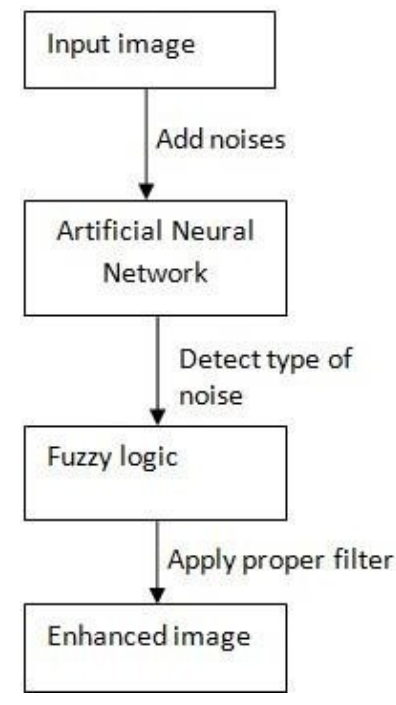

Fig. 2

\section{E. Restoration Algorithm}

Restoration algorithm is used for restoring of images that are corrupt or noisy and estimating the clean, original image. Image restoration is performed by reversing the process that blurred the image and such is performed by imaging a point source image, which is called the Point Spread Function(PSF) to restore the image information lost to be blurring process. Image restoration algorithms distinguish themselves from image enhancement methods in that they are based on models for the degrading process and for the ideal 
image. Here, a novel real-timing algorithm for image restoration is being proposed in edge computing. At first, 10 classical functions are being used to determine the population size and maximum repetition times of adhesion fruit fly optimization algorithm. Secondly, TFOA is used to optimize the optimal parameters of least squares support vector regression kernel function, and the error function of image restoration is taken as an adaptive function of TFOA. Thirdly LLSVR algorithm is used to restore the image. Through the comparison and analysis of experiments, the proposed method could be able meet the requirements of the real-time image restoration, and this proposed algorithm can speed up the image restoration and improve the quality of the image.

\section{F. Contrast Stretching}

Contrast stretching is a simple image enhancement technique that attempts to improve the contrast in an image by stretching the range of intensity values it contains to span a desired range of values of the image. To emerge the range of brightness values in an input image the contrast enhancement techniques are to be used, so that the input image can be efficiently displayed in a proper manner desired by the analyst. The level of contrast in an image may vary because of poor illumination or improper setting in the acquisition sensor device. Therefore, there is a need to manipulate the contrast of an image to compensate for difficulties in image acquisition. The motto behind contrast stretching is to increase the dynamic range of the gray levels in the image being processed. The idea is to modify the dynamic range of the grey-levels in the images. Linear Contrast Stretch is the simplest contrast stretch algorithm that stretches the pixel values of a low-contrast image or high contrast image by extending the dynamic range across the whole image spectrum from $0-(\mathrm{L}-1)$.

\section{G. Koschmieder's model}

Koschmieder proposed that visibility is inversely proportional to the extinction coefficient of air, and this model has been widely adopted during the past century because of its efficiency. Using the radiative transfer theory, the authors present a study of general relationship for the law of contrast reduction and point out that the Koschmieder model is only workable to situations when a common-size object can be viewed from tens of kilometers away. By the adapting this model, we can enhance the gradient and contrast of the image.

\section{CONCLUSION}

Images are more important in day to day life of human beings. The quality of image is affected from environmental conditions including their capturing method, storing technique, and representing behavior. Maximum road accidents in India occur due to poor visibility of objects . the fog changes the contrast of object as differed from originality. The overall statement is targeted to analyze the reality be-
TABLE 1. COMPARATIVE ANALYSIS OF IMAGE ENHANCEMENT TECHNIQUES

\begin{tabular}{|c|c|c|}
\hline S.N. & Techniques & Advantages \\
\hline 1 & $\begin{array}{l}\text { Histogram } \\
\text { equalization }\end{array}$ & $\begin{array}{l}\text { This technique is very much simple. The } \\
\text { global histogram equalization can only be } \\
\text { done completely automatically. }\end{array}$ \\
\hline 2 & $\begin{array}{l}\text { Convolutional } \\
\text { Neural } \\
\text { Network }\end{array}$ & $\begin{array}{l}\text { This algorithm can take images as input, } \\
\text { and assign importance to various objects of } \\
\text { the input image. The preprocessing } \\
\text { required in this algorithm is much lower as } \\
\text { compared to other classification algo- } \\
\text { rithms. }\end{array}$ \\
\hline 3 & $\begin{array}{l}\text { Fuzzy Logic } \\
\text { Technique }\end{array}$ & $\begin{array}{l}\text { The fuzzy rule-based approach is a } \\
\text { powerful method for the formulation of an } \\
\text { expert system in a comprehensive way. } \\
\text { Fuzzy logic represents the good } \\
\text { mathematical frameworks to deal with the } \\
\text { uncertainity of the available information. }\end{array}$ \\
\hline 4 & $\begin{array}{l}\text { Neuro Fuzzy } \\
\text { System }\end{array}$ & $\begin{array}{l}\text { The neural networks basically used for } \\
\text { identification of noise using the statistical } \\
\text { parameters whereas fuzzy logic is used for } \\
\text { the enhancement purpose. }\end{array}$ \\
\hline 5 & $\begin{array}{l}\text { Restoration } \\
\text { Algorithm }\end{array}$ & $\begin{array}{l}\text { This algorithm is a process of recovering } \\
\text { the original image by removing noise and } \\
\text { blur from the image. }\end{array}$ \\
\hline 6 & $\begin{array}{l}\text { Contrast } \\
\text { Stretching }\end{array}$ & $\begin{array}{l}\text { Contrast Stretch is the simplest contrast } \\
\text { stretch algorithm that stretches the pixel } \\
\text { values of a low-contrast image or high- } \\
\text { contrast image by extending the dynamic } \\
\text { range across the whole image spectrum. }\end{array}$ \\
\hline 7 & $\begin{array}{l}\text { Koschmieder's } \\
\text { Model }\end{array}$ & $\begin{array}{l}\text { This model is suitable for both color and } \\
\text { gray scale images and is able to perform } \\
\text { image in real time. With this model, one } \\
\text { can recover the original fog-free image. }\end{array}$ \\
\hline
\end{tabular}

hind the foggy images and their restoration. In this paper, the methodology review of different visibility enhancement approaches and contribution of research communities in each of these category is described elaborately. This paper surveys some of the areas where image enhancement is done with the help of some of the mostly used enhancement techniques.

\section{REFERENCES}

[1] Ping-Juei Liu, Shi-Jinn Horng, Jzau-Sheng Lin, "Contrast in Haze Removal:Configurable Contrast enhancement Model Based on Dark Channel Prior," IEEE Transactions on Image Processing, pp.22122227,2018

[2] Zheqi Lin, "Dehazing for Image and Video Using Guided Filter," Open Journal of Applied Sciences, pp.123-127,2012.

[3] R.Ahila Priyadarshini, S.Aruna, "Visibility Enhancement Technique for Hazy Scenes," $20184^{\text {th }}$ International Conference on Electrical Energy Systems, 2018.

[4] Mohammad Javad Abbaspour, Mehran Yazdi, Mohammadali Masnadi-shirazi, " A new fast method for foggy image enhancement," $201624^{\text {th }}$ Iranian Conference on Electrical Engineering, pp.4-179,2016

[5] Ashok Shrivastava, Sanjay Jain, "Single image dehazing based on one dimensional linear filtering and adoptive histogram equalization method," 2016 international Conference on electrical Electronics, and Optimization Techniques, pp.2-235,2016.

[6] S. S. Negi, Y. S Bhandari, "A Hybrid approach to Image Enhancement using Contrast Stretching on Image Sharpening and the analysis of various cases arising using Histogram," International Conference on Recent Advances and Innovations in Engineering, pp.1-6, 2014. 
[7] Seyed Pooya Ehsani, Hojjat Sayed Mousavi, Babak. H. Khalaj, "Chromosome Image Contrast Enhancement Using Adaptive, Iterative Histogram Matching," $7^{\text {th }}$ Iranian Conference on Machine Vision and Image Processing, pp.8-771, 2011.

[8] Che-Lun Hung, Ren-You Yan, Hsiao-His Wang, “ Parallel image dehazing algorithm based on GPU using fuzzy system and hybrid evolution algorithm," $17^{\text {th }}$ IEEE, pp.2-129, 2016.

[9] Kwang Yeon Choi, Kyeong Min Jeong, Byung Cheol Song, "Fog detection for de-fogging of road driving images," IEEE $20^{\text {th }}$ International Conference on Intelligent Transportation Systems, pp.5-278, 2017.
[10] Khairunnisa Hasikin, Nor Ashidi Mat Isa, "Enhancement of the low contrast image using fuzzy set theory," 2012 UKSim 14 International Conference on Computing Modelling and Simulation, pp.26-1010, 2012.

[11] Raju, Ganesamoorthy, Madhu S. Nair, "A new fuzzy-based decision algorithm for high-density impulse noise removal,” pp. 1-17, 2010.

[12] Dong-Liang Peng, Tie-Jun Wu, "A generalized image enhancement algorithm using fuzzy sets and its application," Proceedings. International Conference on Machine Learning and Cybernetics, pp.13-340, 2002. 